\title{
BMJ Open Development of influenza-associated disease burden pyramid in Shanghai, China, 2010-2017: a Bayesian modelling study
}

Jing Li, ${ }^{1,2,3}$ Chunfang Wang, ${ }^{4}$ Luanqi Ruan, ${ }^{5}$ Shan Jin, ${ }^{4}$ Chuchu Ye, ${ }^{5}$ Huiting Yu (D),${ }^{4}$ Weiping Zhu, ${ }^{5}$ Xiling Wang (iD) ${ }^{1,6}$

To cite: Li J, Wang C, Ruan L, et al. Development of influenza-associated disease burden pyramid in Shanghai, China, 2010-2017: a Bayesian modelling study. BMJ Open 2021;11:e047526. doi:10.1136/ bmjopen-2020-047526

- Prepublication history and additional supplemental material for this paper are available online. To view these files, please visit the journal online (http://dx.doi.org/10.1136/ bmjopen-2020-047526)

$\mathrm{JL}$ and CW contributed equally.

Received 02 December 2020 Accepted 24 August 2021
Check for updates

(C) Author(s) (or their employer(s)) 2021. Re-use permitted under CC BY-NC. No commercial re-use. See rights and permissions. Published by BMJ.

For numbered affiliations see end of article.

\section{Correspondence to}

Xiling Wang;

erinwang@fudan.edu.cn and

Weiping Zhu;

wpzhu@pdcdc.sh.cn

\section{ABSTRACT}

Objectives Negative estimates can be produced when statistical modelling techniques are applied to estimate morbidity and mortality attributable to influenza. Based on the prior knowledge that influenza viruses are hazardous pathogens and have adverse health outcomes of respiratory and circulatory disease (R\&C), we developed an improved model incorporating Bayes' theorem to estimate the disease burden of influenza in Shanghai, China, from 2010 to 2017.

Design A modelling study using aggregated data from administrative systems on weekly R\&C mortality and hospitalisation, influenza surveillance and meteorological data. We constrained the regression coefficients for influenza activity to be positive by truncating the prior distributions at zero.

Setting Shanghai, China.

Participants People registered with R\&C deaths (450 298) and hospitalisations (2621 787, from 1 July 2013), and with influenza-like illness (ILI) outpatient visits (342 149) between 4 January 2010 and 31 December 2017. Primary outcome measures Influenza-associated disease burden (mortality, hospitalisation and outpatient visit rates) and clinical severity (outpatient-mortality, outpatient-hospitalisation and hospitalisation-mortality risks).

Results Influenza was associated with an annual average of 15.49 (95\% credibility interval (Crl) 9.06-22.06) excess R\&C deaths, 100.65 (95\% Crl 48.79-156.78) excess R\&C hospitalisations and 914.95 (95\% Crl 798.51-1023.66) excess ILI outpatient visits per 100000 population in Shanghai. $97.23 \%$ and $80.24 \%$ excess R\&C deaths and hospitalisations occurred in people aged $\geq 65$ years. More than half of excess morbidity and mortality were associated with influenza $A(H 3 N 2)$ virus, and its severities were 1.65-fold to 3.54-fold and 1.47-fold to 2.16-fold higher than that for influenza $\mathrm{A}(\mathrm{H} 1 \mathrm{~N} 1)$ and $\mathrm{B}$ viruses, respectively.

Conclusions The proposed Bayesian approach with reasonable prior information improved estimates of influenza-associated disease burden. Influenza A(H3N2) virus was generally associated with higher morbidity and mortality, and was relatively more severe compared with influenza $A(\mathrm{H} 1 \mathrm{~N} 1)$ and $B$ viruses. Targeted influenza prevention and control strategies for the elderly in Shanghai may substantially reduce the disease burden.

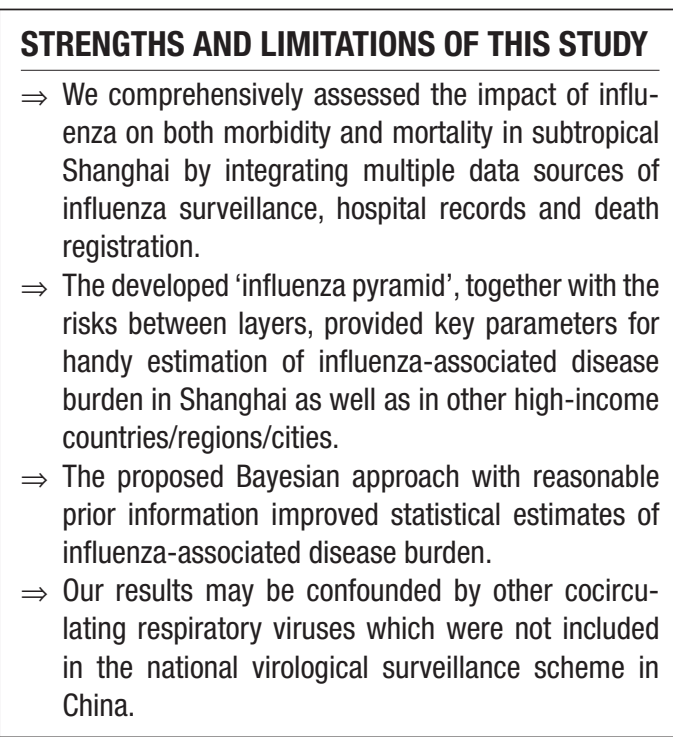

\section{INTRODUCTION}

Annual influenza epidemics result in substantial morbidity and mortality. Influenza virus infections can cause a wide spectrum of diseases, from mild to severe illness requiring hospitalisation, and at times even death. ${ }^{1-4}$ The influenza burden can be presented using the disease pyramid with multiple tiers, including asymptomatic infections, non-medically attended illnesses, medically attended illnesses, hospitalisations, intensive care unit admissions and deaths. ${ }^{5}$ The 'influenza pyramid' provides a full perspective of the disease burden associated with influenza and allows identification of relationships between each layer of the outcome. However, the construction of the pyramid may require merging data gathered from different surveillance systems. Considering the availability of time series data, very few studies have attempted to assess the overall burden of influenza and the 
estimation tended to focus on only one or two particular levels of the pyramid. ${ }^{6-9}$

The disease burden of influenza is difficult to quantify directly because influenza infections are rarely laboratoryconfirmed, or specifically coded as influenza-related in hospital discharge records or death certificates. Statistical modelling techniques have been developed to estimate morbidity and mortality attributable to influenza. ${ }^{3} 1011$ Pneumonia and influenza (P\&I), respiratory and circulatory disease (R\&C) and all-cause are often chosen as outcome variables in modelling methods. P\&I is deemed to be the most specific outcome measure, but this category excludes other respiratory and circulatory diseases exacerbated by influenza. All-cause is too broad and sacrifices specificity; thus, R\&C optimises the balance between sensitivity and specificity. ${ }^{12-14}$

Negative estimates can be produced when statistical modelling techniques are applied to estimate morbidity and mortality attributable to influenza. ${ }^{15-17}$ Based on the prior knowledge that influenza viruses are hazardous pathogens and have adverse health outcomes of R\&C, ${ }^{3418}$ we have developed an improved model incorporating Bayes' theorem to construct the pyramid of influenzaassociated disease burden. We presented the estimates of influenza-associated deaths and hospitalisations due to $\mathrm{R} \& \mathrm{C}$ and influenza-like illness (ILI) outpatient visits in Shanghai, China, from 2010 to 2017.

\section{METHODS}

\section{Influenza surveillance data}

Weekly ILI surveillance and influenza virological surveillance data from 2010 to 2017 were obtained from the Pudong New Area Center for Disease Control and Prevention. Four hospitals conducted year-round surveillance of influenza, including two national sentinel hospitals (Dongfang Hospital and Zhoupu Hospital) and two municipal sentinel hospitals (Pudong Hospital and Eastern Division of Renji Hospital) in Pudong New Area, Shanghai. As we do not have access to raw influenza surveillance data in Shanghai, we used influenza virus activity in Pudong New Area instead. There was a strong correlation of the weekly positive proportion of laboratory-confirmed influenza (LAB\%) between the two data sources from 2010 to $2015(\mathrm{r}=0.80-0.93$, all $\mathrm{p}<0.001)$ (online supplemental figure S1). Weekly LAB\% data in Shanghai were digitised from the time series plot of influenza activity in published literature ${ }^{19}$ using the WebPlotDigitizer software (https:/ / automeris.io/WebPlotDigitizer). ILI surveillance data included weekly numbers of total outpatient visits and age-specific ILI consultations $(0-4,5-14,15-24,25-59$ and $\geq 60$ years). As the elderly people with an age cutpoint of $\geq 65$ years were used in our study, we reclassified these five age groups into $0-14,15-64$ and $\geq 65$ years according to the age distribution of the permanent population in Pudong New Area. Influenza virological surveillance data included weekly numbers of total specimens tested and specimens positive for influenza $\mathrm{A}(\mathrm{H} 1 \mathrm{~N} 1)$ (referring to the 2009 pandemic strain $\mathrm{A}(\mathrm{H} 1 \mathrm{~N} 1)$ pdm09), $\mathrm{A}(\mathrm{H} 3 \mathrm{~N} 2)$ and $B$ viruses.

\section{Mortality and hospitalisation data}

The death registration system of Shanghai is based on its household registration (Hukou) system. ${ }^{20}$ We obtained the weekly mortality data for registered residents in Shanghai for the years 2010-2017 from the Shanghai Center for Disease Control and Prevention. Weekly inpatient data from 2013 to 2017 were obtained from the Shanghai Municipal Health and Family Planning Commission. All medical institutions in Shanghai are required to apply the new edition of the inpatient medical record home page where full indicators were collected since 1 January 2013. We excluded the unstable data for the first half of the year (from the 1st week of 2013 to the 26th week of 2013) during the transition period between the new and old editions of inpatient electronic medical record. We only included inpatients from secondary and tertiary hospitals which had higher data quality than primary hospitals, and they accounted for more than $90 \%$ of the total inpatients in Shanghai. Shanghai provided medical treatments to many patients seeking medical care from other provinces; we only used the data of Shanghai residents for representativeness. Hospital admissions were greatly reduced around the traditional Chinese Spring Festival and National Day, which cannot reflect patients' true healthcare needs, and thus we replaced the week containing the holidays with the average value of the previous and next weeks. ${ }^{21}$

We retrieved underlying causes of death and primary discharge diagnoses coded as respiratory disease (J00J99), circulatory disease (I00-I99) and R\&C (J00-J99 and I00-I99) in the International Classification of Diseases Codes V.10. We assumed that the excess R\&C deaths or hospitalisations would approximate the totality of the influenza-associated deaths or hospitalisations. Data for death and hospitalisation were stratified by age groups ( $<65$ and $\geq 65$ years; $0-5,6-17,18-64$ and $\geq 65$ years, respectively).

\section{Population and meteorological data}

Age-specific annual population sizes, including registered population and permanent population, were obtained from the Shanghai Statistical Yearbook ${ }^{22}$ and National Bureau of Statistics of China, respectively. Annual outpatient visits (medicine and paediatrics) were obtained from China's Health and Family Planning Statistical Yearbook. ${ }^{23}$ Daily temperature and dew point temperature data from 2010 to 2017 were obtained from the Shanghai Meteorological Bureau and were averaged into a weekly level. Absolute humidity was derived from temperature and dew point temperature.

\section{Statistical analysis}

Negative binomial regression models were fitted separately to weekly deaths, hospitalisations and ILI outpatient visits by age groups and diseases in a Bayesian framework 
using the Markov Chain Monte Carlo approach. We used $\mathrm{LAB} \%$ as a proxy variable for influenza activity in the models and assumed a multiplicative association between weekly influenza activity and counts of deaths, hospitalisations or ILI outpatient visits, which has been widely applied in previous literature. ${ }^{3424}$ Natural cubic splines of the calendar week and absolute humidity were added to adjust for time-varying confounders. Viral surveillance data lagged by $0-3$ weeks. We selected the dfs and lag weeks based on the minimum deviance information criterion $^{25}$ and curve fitting (online supplemental table $\mathrm{S} 1$, figure S2-S4). We constrained the regression coefficients for influenza activity to be positive by truncating the normal priors at zero as previous studies have demonstrated positive associations between influenza and R\&C deaths, hospitalisations and ILI outpatient visits. ${ }^{3426}$ More details of the statistical model are available in online supplemental appendix S1.

The influenza-associated excess deaths, hospitalisations and ILI outpatient visits were estimated as the difference between the predicted numbers under the model and the baseline numbers when influenza activity proxies were assumed to be zero. The influenza-associated excess mortality rates, hospitalisation rates and ILI outpatient visit rates were estimated as the numbers of excess deaths, hospitalisations and ILI outpatient visits per 100000 population. Age-standardised rates were derived using the World (WHO 2000-2025) Standard Population as the reference. ${ }^{27}$ With the assumption that the proportion of ILI outpatient visits among medicine and paediatric outpatient visits in the surveillance hospitals of Pudong New Area is representative of Shanghai, we extrapolated the influenza-associated ILI outpatient visits in surveillance hospitals to the general population in Shanghai (see details in online supplemental appendix S2). ${ }^{28}$

Each model was run in 60000 Markov Chain Monte Carlo iterations with the first 30000 iterations discarded as burn-in. We estimated the clinical severity of influenza with different influenza virus types/subtypes and age groups, including outpatient-mortality risks, outpatienthospitalisation risks and hospitalisation-mortality risks, as the ratios of influenza-associated excess mortality rates to ILI outpatient visit rates, hospitalisation rates to ILI outpatient visit rates and mortality rates to hospitalisation rates. The $95 \%$ credibility intervals (CrIs) for excess crude and age-standardised rates were based on the samples drawn from the posterior distributions. Estimates of the outpatient-mortality risks, outpatient-hospitalisation risks, hospitalisation-mortality risks and their 95\% CrIs were also based on these posterior samples.

Analyses were performed using the 'rjags' package of $\mathrm{R}$ software V.3.5.2 (R Foundation for Statistical Computing, Vienna, Austria), an interface between R and JAGS software V.4.3.0 ${ }^{29}$

\section{Patient and public involvement}

Patients and the public were not involved in the design, conduct, reporting or dissemination plans of our research.
RESULTS

\section{Descriptive statistics}

During the study period of 2010-2017, Pudong New Area tested 12283 specimens for influenza viruses, of which 409 were positive for influenza $\mathrm{A}(\mathrm{H} 1 \mathrm{~N} 1)$ virus, 1427 for influenza $\mathrm{A}(\mathrm{H} 3 \mathrm{~N} 2)$ virus and 812 for influenza $\mathrm{B}$ virus (table 1). Influenza B virus was the predominant circulating virus in 2010-2011, and shifted to A(H3N2) virus in subsequent years (figure 1). In general, R\&C mortality rates, R\&C hospitalisation rates and ILI outpatient visit rates increased from 2010 to 2017 in Shanghai, with annual average rates of 392.32, 2407.98 and 5247.92 per 100000 population, respectively (table 1 ).

\section{Influenza-associated disease burden}

During the study period, we estimated an annual average of 2223 (95\% CrI 1300-3164) excess R\&C deaths, 24353 (95\% CrI 11805-37 934) excess R\&C hospitalisations and 218733 (95\% CrI 190 897-244722) excess ILI outpatient visits, attributable to influenza in Shanghai, corresponding to $3.95 \%, 4.18 \%$ and $17.43 \%$ of all R\&C deaths, $\mathrm{R} \& \mathrm{C}$ hospitalisations and ILI outpatient visits, respectively. Accordingly, the estimated crude and age-standardised rates per 100000 population were 15.49 (95\% CrI 9.0622.06) and 7.36 (4.76-10.25) excess R\&C deaths, 100.65 (95\% CrI 48.79-156.78) and 97.90 (70.74-129.02) excess R\&C hospitalisations, and 914.95 (95\% CrI 798.511023.66) and 974.65 (95\% CrI 855.22-1100.21) excess ILI outpatient visits, respectively (table 2). The pyramids of influenza-associated disease burden in Shanghai are shown in figure 2.

Influenza-associated $\mathrm{R} \& \mathrm{C}$ mortality rate for people aged $\geq 65$ years was 81.03 (95\% CrI 50.41-116.99) per 100000 population, which was substantially higher than that for those aged $<65$ years $(0.70,95 \%$ CrI $0.23-1.37$ per 100000 population) (table 2). The age-specific influenzaassociated R\&C hospitalisation rates per 100000 population showed a J-shaped pattern: highest among people aged $\geq 65$ years $(696.38,95 \%$ CrI 396.31-1026.38), second highest among children aged 0-5 years (123.21, 95\% CrI 46.56-212.08), followed by children aged 6-17 years (69.19, 95\% CrI 46.65-91.48) and lowest among people aged 18-64 years (20.73, 95\% CrI 7.02-39.76). Children aged 0-14 years had the highest rate of influenzaassociated ILI outpatient visits (1430.91, 95\% CrI 1096.85-1773.40 per 100000 population), followed by people aged $\geq 65$ (1096.79, 95\% CrI 914.25-1261.59 per 100000 population) and $15-64$ years $(781.92,95 \% \mathrm{CrI}$ $664.68-894.82$ per 100000 population). Among people aged $\geq 65$ years, $97.23 \%$ of excess R\&C deaths and $80.24 \%$ of excess R\&C hospitalisations occurred, whereas of excess ILI outpatient visits, only $13.13 \%$ occurred in this age group.

Influenza $\mathrm{A}(\mathrm{H} 3 \mathrm{~N} 2)$ virus was generally associated with the highest rates of influenza infections, with all-age annual average rates of 10.69 (95\% CrI 5.42-15.80) R\&C deaths, 60.96 (95\% CrI 19.95-100.85) R\&C hospitalisations and 424.74 (95\% CrI 340.90-510.55) ILI outpatient 
Table 1 Annual summary of influenza activity, ILI outpatient visit rate, hospitalisation rate, and mortality rate in Shanghai, 2010-2017

\begin{tabular}{|c|c|c|c|c|c|c|c|c|}
\hline & 2010 & 2011 & 2012 & 2013 & 2014 & 2015 & 2016 & 2017 \\
\hline Influenza A(H1N1) (\%) & $30(17.24)$ & $54(40.00)$ & $0(0.00)$ & $32(19.88)$ & $56(15.34)$ & $68(14.17)$ & $138(30.53)$ & $31(5.09)$ \\
\hline Influenza A(H3N2) (\%) & $45(25.86)$ & $1(0.74)$ & 155 (56.99) & $128(79.50)$ & $220(60.27)$ & $272(56.67)$ & $226(50.00)$ & $380(62.40)$ \\
\hline Total specimens tested & 974 & 755 & 1026 & 1231 & 1935 & 2127 & 2093 & 2142 \\
\hline ILI consultation rate* & 26.73 & 22.31 & 20.99 & 23.00 & 23.94 & 25.85 & 25.00 & 32.18 \\
\hline $\mathrm{R} \& \mathrm{C}$ & - & - & - & 1897.31 & 2131.63 & 2396.44 & 2557.96 & 2801.40 \\
\hline Respiratory disease & - & - & - & 672.00 & 776.21 & 850.69 & 877.10 & 971.29 \\
\hline Circulatory disease & - & - & - & 1225.31 & 1355.42 & 1545.75 & 1680.86 & 1830.12 \\
\hline \multicolumn{9}{|l|}{ Mortality rate§ } \\
\hline Registered population & 14123202 & 14193600 & 14269319 & 14323391 & 14386900 & 14429676 & 14499990 & 14551300 \\
\hline Permanent population & 23019196 & 23474591 & 23804303 & 24151500 & 24256797 & 24152700 & 24197001 & 24197001 \\
\hline \multicolumn{9}{|c|}{$\begin{array}{l}\text { Influenza surveillance data were recorded in Pudong New Area, including specimens positive for influenza by type/subtype, total specimens tested, } \\
\text { ILI consultations and total outpatient visits } \\
\text { *ILI consultation rate per } 1000 \text { outpatient visits. } \\
\text { †ILI outpatient visit rate per } 100000 \text { permanent population. } \\
\text { fHospitalisation rate per } 100000 \text { permanent population. } \\
\text { §Mortality rate per } 100000 \text { registered population. } \\
\text { ILI, influenza-like illness; R\&C, respiratory and circulatory disease. }\end{array}$} \\
\hline
\end{tabular}

visits per 100000 population, respectively (table 2 and figure 2). When broken down by diseases, for all ages and $\geq 65$ years, the influenza-associated mortality rates for respiratory disease $(6.90,95 \%$ CrI $5.27-8.39$, and 35.70 , 95\% CrI 27.78-44.16, per 100000 population) were 1.40-fold to 1.48-fold lower than those for circulatory disease $(9.64,95 \% \mathrm{CrI} 5.05-14.51$, and $52.81,95 \%$ CrI 28.66-79.37, per 100000 population), while the influenza-associated hospitalisation rates for respiratory disease $(72.48,95 \%$ CrI 51.34-95.11, and 501.90, 95\% CrI 406.51-600.23, per 100000 population) were 2.39-fold to 2.40-fold higher than those for circulatory disease (30.20, 95\% CrI 9.61-60.12, and 210.20, 95\% CrI 63.08-404.70) per 100000 population) (table 2). A similar J-shaped age pattern was also observed in influenza-associated respiratory hospitalisation rates, while for circulatory hospitalisation, the estimated rates increased with age.

\section{Influenza-associated clinical severity}

Influenza-associated outpatient-mortality risk, outpatient-hospitalisation risk and hospitalisation-mortality risk were estimated to be $1.69 \%$ (95\% CrI $0.97 \%-2.48 \%$ ),

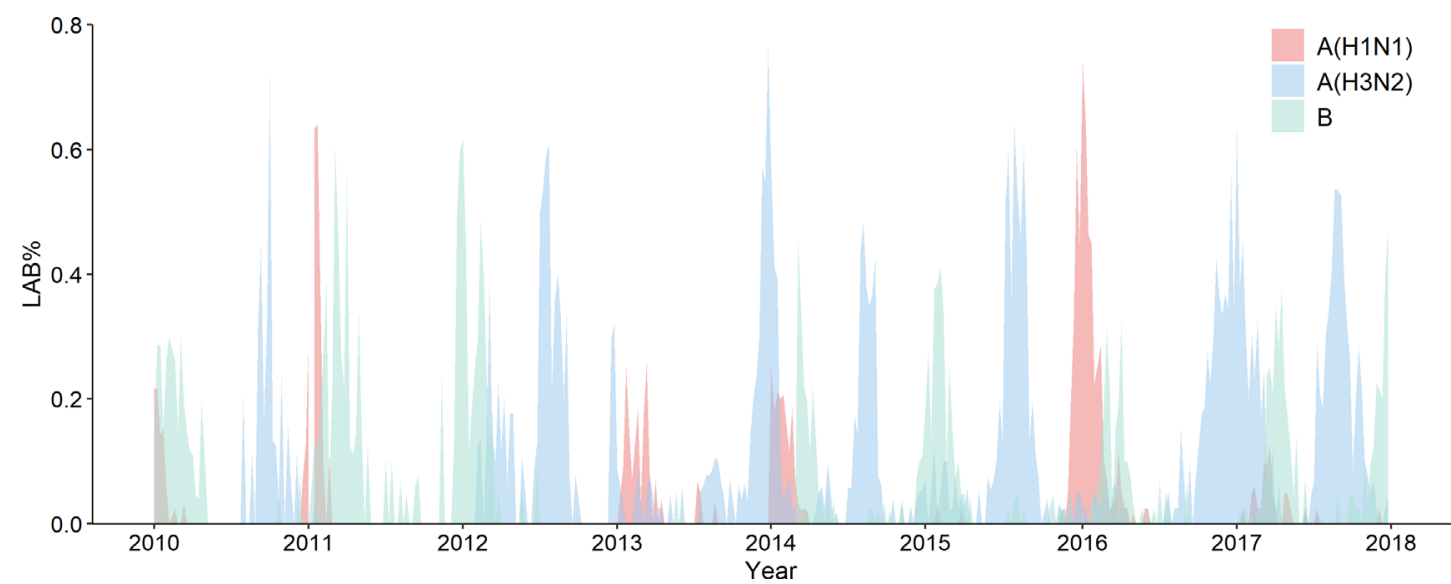

Figure 1 Influenza activity by type/subtype in Pudong New Area, Shanghai, 2010-2017. LAB\%, weekly positive proportion of laboratory-confirmed influenza. 
Table 2 Mean annual influenza-associated excess mortality rates and hospitalisation rates due to R\&Cs, and ILI outpatient visit rates (per 100000 population) by influenza type/subtype and age group in Shanghai, 2010-2017

\begin{tabular}{|c|c|c|c|c|}
\hline & All influenza & $A(H 1 N 1)$ & $A(H 3 N 2)$ & B \\
\hline \multicolumn{5}{|c|}{ Influenza-associated excess mortality rate } \\
\hline \multicolumn{5}{|l|}{$\mathrm{R} \& \mathrm{C}$} \\
\hline Age-standardised & $7.36(4.76-10.25)$ & $1.07(0.27-2.21)$ & $4.97(2.90-7.17)$ & $1.31(0.21-3.09)$ \\
\hline All ages & $15.49(9.06-22.06)$ & $2.10(0.17-4.66)$ & $10.69(5.42-15.80)$ & $2.62(0.16-6.86)$ \\
\hline$<65$ years & $0.70(0.23-1.37)$ & $0.26(0.02-0.57)$ & $0.22(0.01-0.74)$ & $0.16(0.01-0.59)$ \\
\hline$\geq 65$ years & $81.03(50.41-116.99)$ & $9.73(0.86-23.83)$ & $57.47(31.71-82.58)$ & $13.62(0.93-34.48)$ \\
\hline \multicolumn{5}{|l|}{ Respiratory disease } \\
\hline Age-standardised & $3.40(2.73-4.13)$ & $0.65(0.37-0.95)$ & $2.16(1.70-2.61)$ & $0.65(0.20-1.09)$ \\
\hline All ages & $6.90(5.27-8.39)$ & $1.16(0.53-1.83)$ & $4.50(3.41-5.51)$ & $1.29(0.27-2.39)$ \\
\hline$<65$ years & $0.51(0.34-0.69)$ & $0.18(0.10-0.26)$ & $0.27(0.14-0.40)$ & $0.06(0-0.18)$ \\
\hline$\geq 65$ years & $35.70(27.78-44.16)$ & $5.90(2.49-9.39)$ & $23.13(18.04-27.84)$ & $7.21(1.71-12.58)$ \\
\hline \multicolumn{5}{|l|}{ Circulatory disease } \\
\hline Age-standardised & $4.82(2.81-7.04)$ & $0.78(0.16-1.66)$ & $3.18(1.41-4.76)$ & $0.77(0.10-1.99)$ \\
\hline All ages & $9.64(5.05-14.51)$ & $1.43(0.10-3.59)$ & $6.56(2.64-10.66)$ & $1.48(0.07-4.35)$ \\
\hline$<65$ years & $0.50(0.17-1.01)$ & $0.20(0.02-0.47)$ & $0.14(0.01-0.56)$ & $0.11(0.01-0.44)$ \\
\hline$\geq 65$ years & $52.81(28.66-79.37)$ & $7.00(0.31-17.29)$ & $36.78(15.03-56.76)$ & $7.78(0.36-22.57)$ \\
\hline \multicolumn{5}{|c|}{ Influenza-associated excess hospitalisation rate } \\
\hline \multicolumn{5}{|l|}{$\mathrm{R} \& \mathrm{C}$} \\
\hline Age-standardised & $97.90(70.74-129.02)$ & $19.89(9.82-31.10)$ & $51.22(30.34-73.87)$ & $26.69(14.29-43.22)$ \\
\hline All ages & 100.65 (48.79-156.78) & $19.89(2.38-38.93)$ & $60.96(19.95-100.85)$ & $20.33(1.62-53.10)$ \\
\hline $0-5$ years & $123.21(46.56-212.08)$ & $38.04(8.59-70.90)$ & $38.19(1.49-100.54)$ & $44.47(4.86-93.90)$ \\
\hline $6-17$ years & $69.19(46.65-91.48)$ & $14.13(5.24-22.53)$ & $19.49(2.93-37.55)$ & $36.15(24.95-48.42)$ \\
\hline $18-64$ years & $20.73(7.02-39.76)$ & $5.87(0.54-12.78)$ & $10.02(0.73-24.30)$ & $4.24(0.21-12.87)$ \\
\hline$\geq 65$ years & 696.38 (396.31-1026.38) & 110.79 (14.68-237.24) & $440.96(208.84-687.51)$ & $140.10(11.93-326.64)$ \\
\hline \multicolumn{5}{|l|}{ Respiratory disease } \\
\hline Age-standardised & 84.22 (70.58-100.36) & 20.08 (13.89-25.93) & $45.87(34.88-58.36)$ & 19.93 (12.20-28.69) \\
\hline All ages & 72.48 (51.34-95.11) & $14.46(5.63-23.41)$ & $47.89(29.01-66.75)$ & $10.52(1.13-23.15)$ \\
\hline $0-5$ years & 171.40 (78.58-277.30) & 50.95 (15.35-84.07) & $78.11(9.17-159.17)$ & 40.15 (4.60-94.86) \\
\hline $6-17$ years & 77.07 (50.02-103.71) & 18.88 (7.59-29.43) & 26.72 (7.02-47.51) & 32.95 (20.01-45.33) \\
\hline $18-64$ years & $15.46(8.98-22.11)$ & $4.31(1.69-6.94)$ & $8.77(3.78-13.74)$ & $2.31(0.21-5.76)$ \\
\hline$\geq 65$ years & $501.90(406.51-600.23)$ & $97.86(53.77-146.64)$ & $323.59(243.86-400.31)$ & $87.29(22.88-153.14)$ \\
\hline \multicolumn{5}{|l|}{ Circulatory disease } \\
\hline Age-standardised & $23.42(10.38-41.41)$ & $4.77(1.06-11.18)$ & $10.94(2.56-23.71)$ & $6.96(1.42-16.36)$ \\
\hline All ages & 30.20 (9.61-60.12) & $6.10(0.40-17.24)$ & $14.23(0.88-36.60)$ & $8.55(0.47-24.98)$ \\
\hline $0-5$ years & $1.03(0.30-2.32)$ & $0.17(0.01-0.68)$ & $0.36(0.02-1.51)$ & $0.35(0.01-1.24)$ \\
\hline $6-17$ years & $2.30(0.91-3.98)$ & $0.78(0.14-1.44)$ & $0.93(0.09-2.25)$ & $0.54(0.04-1.41)$ \\
\hline $18-64$ years & $8.44(2.28-19.89)$ & $1.66(0.06-5.78)$ & $4.23(0.19-13.01)$ & $1.89(0.09-7.35)$ \\
\hline$\geq 65$ years & 210.20 (63.08-404.70) & $41.37(2.78-114.16)$ & $95.80(6.27-237.28)$ & $65.85(4.36-173.23)$ \\
\hline \multicolumn{5}{|c|}{ Influenza-associated excess ILI outpatient visit rate } \\
\hline Age-standardised & $974.65(855.22-1,100.21)$ & $332.17(292.83-375.69)$ & $414.98(329.49-505.67)$ & $256.89(186.71-326.45)$ \\
\hline All ages & $914.95(798.51-1,023.66)$ & $295.36(253.86-338.55)$ & $424.74(340.90-510.55)$ & $224.37(158.26-284.61)$ \\
\hline $0-14$ years & $1430.91(1,096.85-1,773.40)$ & $482.20(370.05-605.77)$ & $382.26(156.64-607.09)$ & $586.25(360.05-818.06)$ \\
\hline $15-64$ years & $781.92(664.68-894.82)$ & $284.84(240.37-328.79)$ & $389.71(299.27-480.25)$ & $134.60(76.48-196.58)$ \\
\hline$\geq 65$ years & $1096.79(914.25-1,261.59)$ & $228.14(169.99-281.51)$ & 722.65 (575.04-868.30) & $177.45(88.86-268.13)$ \\
\hline \multicolumn{5}{|c|}{ ILI, influenza-like illness; R\&C, respiratory and circulatory disease. } \\
\hline
\end{tabular}




\section{A. All ages}

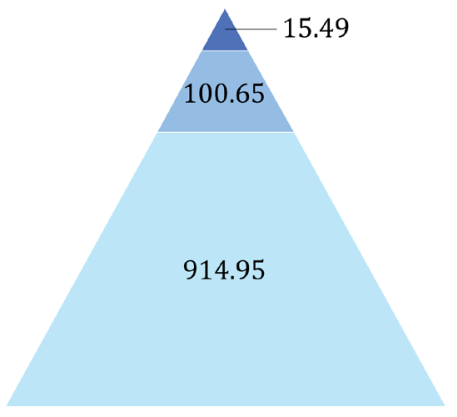

All influenza
Influenza-associated $\mathrm{R} \& \mathrm{C}$ mortality rate (per 100,000 population) Influenza-associated $R \& C$ hospitalisation rate (per 100,000 population) Influenza-associated ILI outpatient visit rate (per 100,000 population)

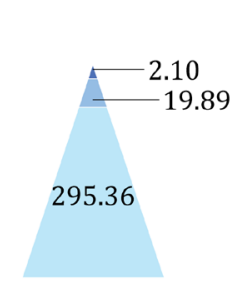

$\mathrm{A}(\mathrm{H} 1 \mathrm{~N} 1)$

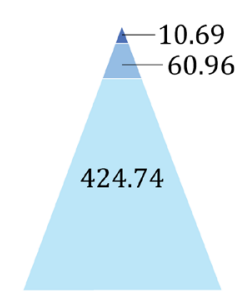

A(H3N2)

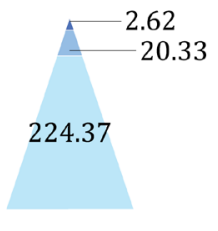

B

\section{B. Age $\geq 65$ years}

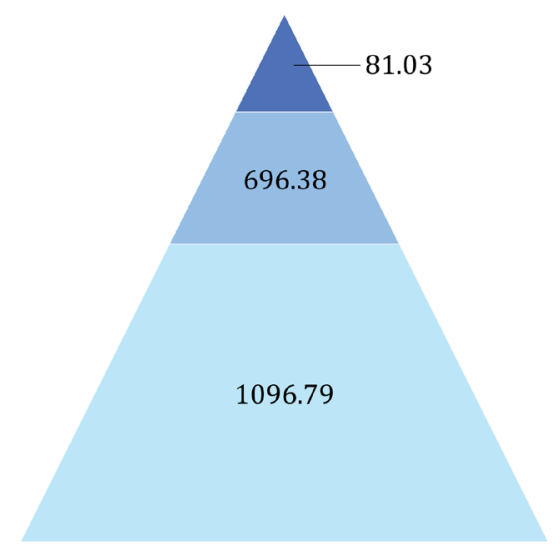

All influenza

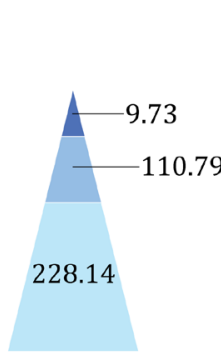

$\mathrm{A}(\mathrm{H} 1 \mathrm{~N} 1)$

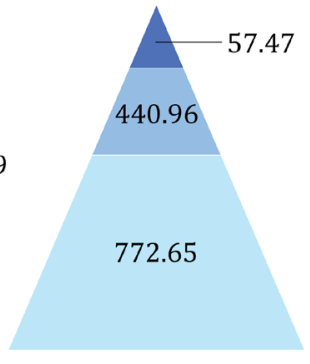

$\mathrm{A}(\mathrm{H} 3 \mathrm{~N} 2)$

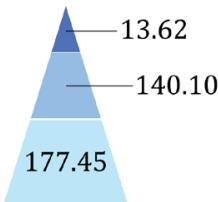

B

Figure 2 Pyramid of influenza burden by influenza virus types/subtypes and age groups in Shanghai, 2010-2017. ILI, influenza-like illness; R\&C, respiratory and circulatory disease.

$11.09 \%(95 \%$ CrI $5.23 \%-17.39 \%)$ and $15.29 \%(95 \%$ CrI $7.68 \%-34.85 \%$ ), respectively (online supplemental table S2); that is to say, we estimated that there were 1.69 excess $\mathrm{R} \& \mathrm{C}$ deaths and 11.09 excess $\mathrm{R} \& \mathrm{C}$ hospitalisations for every 100 excess ILI outpatient visits, and 15.29 excess R\&C deaths for every 100 excess R\&C hospitalisations in Shanghai. The estimated risks for people aged $\geq 65$ years were higher than those for all ages except for hospitalisation-mortality risks (figure 3). When comparing these risks between different types/subtypes of influenza viruses, we found that influenza $\mathrm{A}(\mathrm{H} 3 \mathrm{~N} 2)$ virus had the greatest outpatient-mortality risks, outpatient-hospitalisation risks and hospitalisation-mortality risks across different age groups. Its severities were 1.65-fold to 3.54fold and 1.47-fold to 2.16-fold higher than those for influenza $\mathrm{A}(\mathrm{H} 1 \mathrm{~N} 1)$ and $\mathrm{B}$ viruses, respectively (online supplemental table S2). However, the difference did not reach statistical significance. Also, we presented separately
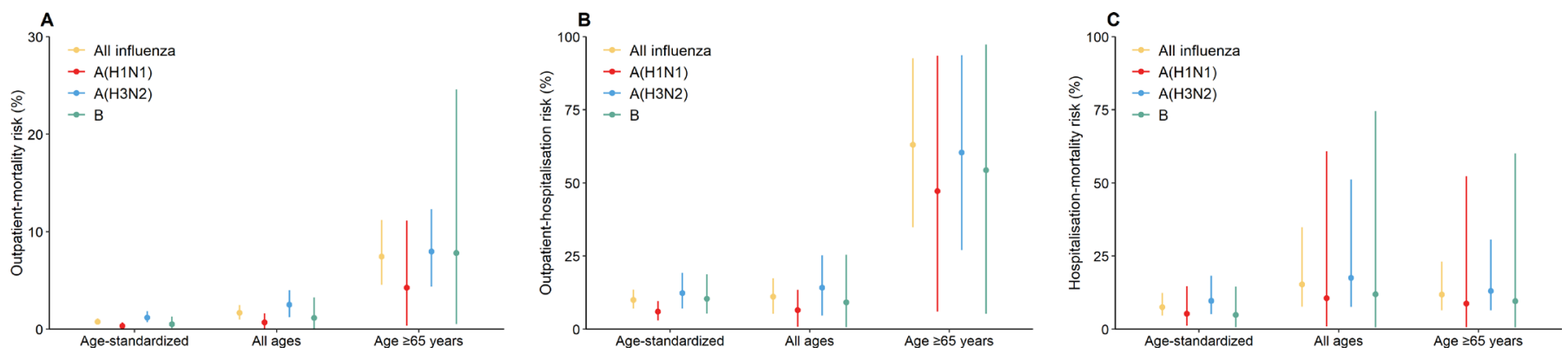

Figure 3 Clinical severity of influenza with different influenza virus types/subtypes and age groups. (A) Outpatient-mortality risk, (B) outpatient-hospitalisation risk and (C) hospitalisation-mortality risk. 
the influenza-associated hospitalisation-mortality risks for respiratory disease and circulatory disease (online supplemental figure S5). People with the circulatory disease had a 3.22-fold higher hospitalisation-mortality risk than those with respiratory disease (online supplemental table S2).

\section{DISCUSSION}

Although there were great year-to-year variations in influenza-associated mortality and morbidity from 2010 to 2017, we estimated that influenza contributed to an annual average of 15.49 (95\% CrI 9.06-22.06) excess R\&C deaths, 100.65 (95\% CrI 48.79-156.78) excess R\&C hospitalisations and 914.95 (95\% CrI 798.51-1023.66) excess ILI outpatient visits per 100000 population in Shanghai. Our crude or age-standardised estimates of influenza-associated mortality and morbidity are comparable with the corresponding estimates published for the USA (R\&C death: 9.9 per 100000 population, ${ }^{30} \mathrm{R} \& \mathrm{C}$ hospitalisation: 88.4 per 100000 population ${ }^{4}$ and ILI outpatient visit: $755-870$ per 100000 population) ${ }^{31}{ }^{32}$; Argentina (R\&C death: 21.3 per 100000 population and R\&C hospitalisation: 57 per 100000 population $)^{14}$; Hong Kong SAR (R\&C death: 7.7 per 100000 population) ${ }^{33}$; and five southern Chinese cities (R\&C death: 8.8 per 100000 population). ${ }^{34}$ In particular, Hong Kong SAR reported an approximately twofold higher hospitalisation burden than our estimates, ${ }^{33}$ which could be explained by the result of expanding health service capacities and lowering admission criteria due to the ageing population of Hong Kong in recent years. ${ }^{13} 245$ However, caution is needed when comparing these estimates between countries/regions/cities due to variations in the study period, predominant circulating influenza virus, age structure of population, influenza vaccination coverage and statistical model used. Influenza A(H3N2) virus accounted for the highest disease burden than the other two types, which has also been demonstrated by other studies. ${ }^{4146}$ Influenza $\mathrm{A}(\mathrm{H} 3 \mathrm{~N} 2)$ virus is believed to have more frequent antigen drift and higher virulence than seasonal influenza A(H1N1) and B viruses. ${ }^{37} 38$ Additionally, our study revealed that influenza $\mathrm{A}(\mathrm{H} 3 \mathrm{~N} 2)$ virus was relatively more severe as well.

The elderly people were most severely affected by influenza, with the highest influenza-associated mortality and hospitalisation rates, and the second highest influenzaassociated ILI outpatient visit rates. The ageing of the Shanghai population is an important contributor to the high rates of influenza-associated mortality and morbidity. For example, the influenza-associated mortality rates after age standardisation decreased by more than half. Between 2010 and 2017, the registered population aged $\geq 65$ years in Shanghai increased by $28.70 \%$, of about 3.18 million by 2017 , accounting for $21.83 \%$ of the total population. Interestingly, people aged $\geq 65$ years had lower hospitalisation-mortality risks than for all ages. A possible explanation might be that young adults are less likely to be hospitalised compared with elderly people, but some of them do develop serious illnesses and require hospitalisation, and thus have a greater risk of death.

Currently, population-based studies of influenzaassociated mortality and morbidity have been increasingly reported in mainland China. Of these, two nationally representative studies estimated province-level influenzaassociated respiratory mortality rates and ILI outpatient visit rates. However, studies on influenza-associated hospitalisation burden were limited to Beijing, ${ }^{39}{ }^{40} \mathrm{Jiangsu}^{41-45}$ and Hubei. ${ }^{46}$ Our study comprehensively assessed the impact of influenza in Shanghai, especially the hospitalisation burden, which has never been assessed before. The estimated ratios between each layer of the influenza pyramid provided key parameters for handy estimation of influenza-associated disease burden in Shanghai as well as in other high-income countries/regions/cities.

Performing regression analyses in a Bayesian framework had two advantages in our study. First, positive priors truncated the posterior distributions of regression coefficients for influenza activity to ensure the positivity of the generated samples. Otherwise, it was difficult to explain that influenza was protective to human health. Second, Bayesian posterior samples allowed us to estimate the $95 \%$ CrIs for these ratios, which could be challenging in a frequentist framework. Various proxies for influenza activity have been developed, and the most commonly used proxy variable was LAB\%. ${ }^{47} \mathrm{Yu}$ et $a l^{19}$ estimated influenza-associated P\&I mortality, considering three different proxies for influenza activity: LAB number (positive number of laboratory-confirmed influenza), $\mathrm{LAB} \%$ and $\mathrm{LAB} \% \times \mathrm{ILI} \%$ (the product of positive proportion of laboratory-confirmed influenza and ILI consultation rate). The results of this study demonstrated that $\mathrm{LAB} \%$ proxy produced slightly higher estimates of influenza-associated mortality than LAB number and LAB $\% \times$ ILI $\%$ proxies. Another study by Chan $e t a t^{48}$ evaluated the performance of various virus proxy variables in estimating excess hospitalisation and concluded that LAB \% $\times$ ILI\% proxy provided more reliable estimates if age-specific virus data were not available. However, in general, different influenza activity proxies provided comparable estimates for influenza disease burden. We used LAB\% proxy in this study which has been adopted to link influenza and health outcomes in previous literature. $^{34} 49$

Nevertheless, our study had several limitations. First, despite a strong correlation of influenza virus activity between Pudong New Area and Shanghai, LAB\% in Shanghai was slightly higher than that in Pudong New Area (median difference: 6.12\%). Replacement of influenza activity in Pudong New Area may lead to an underestimation of the influenza burden in Shanghai from 2010 to 2017. Second, national ILI surveillance data were recorded by five predefined age groups; thus, influenza burden estimates for ILI outpatient visits and hospitalisations were difficult to compare across different age groups. Additionally, when extrapolating influenza-associated 
ILI outpatient visits and reclassifying ILI consultations, we were based on representativeness assumptions, but further evaluation may be needed. Third, R\&C was chosen to optimise sensitivity-specificity balance, but influenza-associated R\&C events may be slightly lower than the totality of the influenza-associated events. Fourth, we excluded hospitalisation data of inpatients from primary hospitals and with non-Shanghai residential addresses, considering the study's quality and population representativeness. The former may underestimate and the latter may overestimate the influenza-associated hospitalisation burden. Fifth, our estimates of influenzaassociated mortality and morbidity burden were not exactly based on the same population (registered population and permanent population respectively). Deaths of permanent population without Shanghai Hukou are usually registered at their place of household registration. Using the permanent population as the denominator may underestimate the influenza-associated mortality burden. Finally, we did not have data on cocirculating respiratory viruses, such as respiratory syncytial virus, which may have confounded the results.

In conclusion, the proposed Bayesian approach with reasonable prior information improved estimates of influenza-associated disease burden. Our study highlighted the substantial morbidity and mortality burden attributed to influenza in Shanghai. Influenza A(H3N2) virus was generally associated with more morbidity and mortality, and was relatively more severe compared with influenza $\mathrm{A}(\mathrm{H} 1 \mathrm{~N} 1)$ and $\mathrm{B}$ viruses. Targeted influenza prevention and control strategies for the elderly in Shanghai may substantially impact the disease burden.

\section{Author affiliations}

${ }^{1}$ School of Public Health, Fudan University, Shanghai, Shanghai, China

${ }^{2}$ Renal Division, Peking University Shenzhen Hospital, Shenzhen, Guangdong, China ${ }^{3}$ Clinical Research Academy, Peking University Shenzhen Hospital, Shenzhen,

Guangdong, China

${ }^{4}$ Department of Vital Statistics, Shanghai Municipal Center for Disease Control and Prevention, Shanghai, China

${ }^{5}$ Research Base of Key Laboratory of Surveillance and Early Warning on Infectious Disease, Pudong New Area Center for Disease Control and Prevention, Shanghai, China

${ }^{6}$ Shanghai Key Laboratory of Meteorology and Health, Shanghai, China

Contributors XW and WZ conceived, designed and supervised the study. JL, CW, WZ, LR, SJ, CY and HY participated in data collection. JL, XW and SJ conducted statistical analyses. JL and CW drafted the manuscript. XW and WZ commented on the data and their interpretation, and revised the content critically. All authors read and approved the final manuscript.

Funding XW is supported by the National Nature and Science Foundation of China (grant number 81602936). WZ, CY and LR are supported by the National Science and Technology Major Project (grant number 2018ZX10713001008). The funding sources had no role in the study design, data collection, data analysis or writing of the report.

Competing interests None declared.

Patient consent for publication Not required.

Provenance and peer review Not commissioned; externally peer reviewed.

Data availability statement Data are available upon reasonable request. Data are available upon reasonable request to the corresponding author.
Supplemental material This content has been supplied by the author(s). It has not been vetted by BMJ Publishing Group Limited (BMJ) and may not have been peer-reviewed. Any opinions or recommendations discussed are solely those of the author(s) and are not endorsed by BMJ. BMJ disclaims all liability and responsibility arising from any reliance placed on the content. Where the content includes any translated material, BMJ does not warrant the accuracy and reliability of the translations (including but not limited to local regulations, clinical guidelines, terminology, drug names and drug dosages), and is not responsible for any error and/or omissions arising from translation and adaptation or otherwise.

Open access This is an open access article distributed in accordance with the Creative Commons Attribution Non Commercial (CC BY-NC 4.0) license, which permits others to distribute, remix, adapt, build upon this work non-commercially, and license their derivative works on different terms, provided the original work is properly cited, appropriate credit is given, any changes made indicated, and the use is non-commercial. See: http://creativecommons.org/licenses/by-nc/4.0/.

ORCID iDs

Huiting Yu http://orcid.org/0000-0002-8979-5238

Xiling Wang http://orcid.org/0000-0002-7164-6189

\section{REFERENCES}

1 World Health Organization. Fact sheet on seasonal influenza. Available: https://www.who.int/en/news-room/fact-sheets/detail/ influenza-(seasonal) [Accessed 3 Apr 2020].

2 Tempia S, Walaza S, Moyes J, et al. Quantifying how different clinical presentations, levels of severity, and healthcare attendance shape the burden of influenza-associated illness: a modeling study from South Africa. Clin Infect Dis 2019;69:1036-48.

3 Thompson WW, Shay DK, Weintraub E, et al. Mortality associated with influenza and respiratory syncytial virus in the United States. JAMA 2003;289:179-86.

4 Thompson WW, Shay DK, Weintraub E, et al. Influenza-associated hospitalizations in the United States. JAMA 2004;292:1333-40.

5 Pelat C, Ferguson NM, White PJ, et al. Optimizing the precision of case fatality ratio estimates under the surveillance pyramid approach. Am J Epidemiol 2014;180:1036-46.

6 Menec VH, Black C, MacWilliam L, et al. The impact of influenzaassociated respiratory illnesses on hospitalizations, physician visits, emergency room visits, and mortality. Can J Public Health 2003;94:59-63.

7 Pitman RJ, Melegaro A, Gelb D, et al. Assessing the burden of influenza and other respiratory infections in England and Wales. $J$ Infect 2007;54:530-8.

8 Choi WS, Cowling BJ, Noh JY, et al. Disease burden of 2013-2014 seasonal influenza in adults in Korea. PLoS One 2017;12:e0172012.

9 TroegerCE, BlackerBF, KhalillA, et al. Mortality, morbidity, and hospitalisations due to influenza lower respiratory tract infections, 2017: an analysis for the global burden of disease study 2017. Lancet Respir Med 2019;7:69-89.

10 Chow A, Ma S, Ling AE, et al. Influenza-associated deaths in tropical Singapore. Emerg Infect Dis 2006;12:114-21.

11 Serfling RE. Methods for current statistical analysis of excess pneumonia-influenza deaths. Public Health Rep 1963;78:494-506.

12 Matias G, Taylor RJ, Haguinet F, et al. Modelling estimates of agespecific influenza-related hospitalisation and mortality in the United Kingdom. BMC Public Health 2016;16:481.

13 Li CK, Choi BCK, Wong TW. Influenza-related deaths and hospitalizations in Hong Kong: a subtropical area. Public Health 2006;120:517-24.

14 Azziz-Baumgartner E, Cabrera AM, Cheng P-Y, et al. Incidence of influenza-associated mortality and hospitalizations in Argentina during 2002-2009. Influenza Other Respir Viruses 2013;7:710-7.

15 Wu P, Goldstein E, Ho LM, et al. Excess mortality associated with influenza A and B virus in Hong Kong, 1998-2009. J Infect Dis 2012;206:1862-71.

16 Park M, Wu P, Goldstein E, et al. Influenza-Associated excess mortality in South Korea. Am J Prev Med 2016;50:e111-9.

17 Yang L, Ma S, Chen PY, et al. Influenza associated mortality in the subtropics and tropics: results from three Asian cities. Vaccine 2011;29:8909-14.

18 Matias G, Haguinet F, Lustig RL, et al. Model estimates of the burden of outpatient visits attributable to influenza in the United States. BMC Infect Dis 2016;16:641.

19 Yu X, Wang C, Chen T, et al. Excess pneumonia and influenza mortality attributable to seasonal influenza in subtropical Shanghai, China. BMC Infect Dis 2017;17:756-56. 
20 Zhao J, Tu EJ-C, Law C-K. The incomparability of cause of death statistics under "one country, two systems": Shanghai versus Hong Kong. Popul Health Metr 2017; 15:37.

21 Tang L, Chen P-F, Hu X-Q, et al. Effect of Chinese national holidays and weekends versus weekday admission on clinical outcomes in patients with STEMI undergoing primary PCI. J Geriatr Cardiol 2017;14:604-13.

22 Shanghai Bureau of Statistics. Shanghai statistical yearbook. Available: http://tjj.sh.gov.cn/tjnj/index.html [Accessed 16 Apr 2020].

23 National Health and Family Planning Commission of the People's Republic of China. China's health and family planning statistical yearbook. Available: http://www.nhc.gov.cn/zwgkzt/tjnj/list.shtml [Accessed 16 Apr 2020]

24 Yang L, Wang XL, Chan KP, et al. Hospitalisation associated with the $2009 \mathrm{H} 1 \mathrm{~N} 1$ pandemic and seasonal influenza in Hong Kong, 2005 to 2010. Euro Surveill 2012;17. doi:10.2807/ese.17.45.20309-en. [Epub ahead of print: 08 Nov 2012].

25 Pooley CM, Marion G. Bayesian model evidence as a practical alternative to deviance information criterion. $R$ Soc Open Sci 2018;5:171519.

26 Feng L, Feng S, Chen T, et al. Burden of influenza-associated outpatient influenza-like illness consultations in China, 2006-2015: a population-based study. Influenza Other Respi Viruses 2020;14:162-72.

27 National Cancer Institute. Standard populations - single ages. Available: https://seer.cancer.gov/stdpopulations/stdpop.singleages. html

28 Feng L, Feng S, Chen T, et al. Burden of influenza-associated outpatient influenza-like illness consultations in China, 20062015: a population-based study. Influenza Other Respir Viruses 2020:14:162-72.

29 Plummer M. JAGS: a program for analysis of Bayesian graphical models using Gibbs sampling. in: Proceeding of the 3rd international workshop on distributed statistical computing (DSC 2003), Vienna, Austria 2003;124.

30 Thompson WW, Weintraub E, Dhankhar P, et al. Estimates of US influenza-associated deaths made using four different methods. Influenza Other Respir Viruses 2009;3:37-49.

31 Fowlkes A, Dasgupta S, Chao E, et al. Estimating influenza incidence and rates of influenza-like illness in the outpatient setting. Influenza Other Respir Viruses 2013;7:694-700.

32 Fowlkes A, Steffens A, Temte J, et al. Incidence of medically attended influenza during pandemic and post-pandemic seasons through the influenza incidence surveillance project, 2009-13. Lancet Respir Med 2015;3:709-18.

33 Wu P, Presanis AM, Bond HS, et al. A joint analysis of influenzaassociated hospitalizations and mortality in Hong Kong, 1998-2013. Sci Rep 2017;7:929.
34 Feng L, Shay DK, Jiang Y, et al. Influenza-associated mortality in temperate and subtropical Chinese cities, 2003-2008. Bull World Health Organ 2012;90:279-88.

35 Wong CM, Yang L, Chan KP, et al. Influenza-associated hospitalization in a subtropical city. PLoS Med 2006;3:e121.

36 Zhang $\mathrm{H}$, Xiong Q, Wu P, et al. Influenza-associated mortality in Yancheng, China, 2011-15. Influenza Other Respir Viruses 2018;12:98-103.

37 Ferguson NM, Galvani AP, Bush RM. Ecological and immunological determinants of influenza evolution. Nature 2003;422:428-33.

38 Rambaut A, Pybus OG, Nelson MI, et al. The genomic and epidemiological dynamics of human influenza A virus. Nature 2008;453:615-9.

39 Zhang Y, Muscatello DJ, Wang Q, et al. Hospitalizations for influenzaassociated severe acute respiratory infection, Beijing, China, 20142016. Emerg Infect Dis 2018;24:2098-102.

40 Zhao X, Zhang Y, Yang J. Study of characteristics and estimation of hospitalization rate for hospitalized cases of influenza in Huairou district. International Journal of Virology 2018;25:281-5.

41 Feng L, Fei F, Li S, et al. [Influenza-associated-excess-hospitalization in children, Wuxi city, Jiangsu province, 2005-2010]. Zhonghua Liu Xing Bing Xue Za Zhi 2014;35:699-703.

42 Zhang X, Zhang J, Chen L, et al. Pneumonia and influenza hospitalizations among children under 5 years of age in Suzhou, China, 2005-2011. Influenza Other Respir Viruses 2017;11:15-22.

43 Ji W, Zhang T, Zhang X, et al. The epidemiology of hospitalized influenza in children, a two year population-based study in the people's Republic of China. BMC Health Serv Res 2010;10:82.

$44 \mathrm{Yu}$ J, Zhang X, Shan W, et al. Influenza-associated hospitalization in children younger than 5 years of age in Suzhou, China, 2011-2016. Pediatr Infect Dis J 2019;38:445-52.

45 Zhang W, Yu J, Chen L. Hospitalization rates for influenzaassociated severe acute respiratory illness in children younger than five years old in Suzhou of China, 2016-2018. Chin J Prev Med 2019;53:1056-9.

$46 \mathrm{Yu} \mathrm{H}$, Huang J, Huai Y, et al. The substantial hospitalization burden of influenza in central China: surveillance for severe, acute respiratory infection, and influenza viruses, 2010-2012. Influenza Other Respir Viruses 2014;8:53-65.

47 Li L, Wong JY, Wu P, et al. Heterogeneity in estimates of the impact of influenza on population mortality: a systematic review. Am J Epidemiol 2018;187:378-88.

48 Chan KP, Wong CM, Chiu SSS, et al. A robust parameter estimation method for estimating disease burden of respiratory viruses. PLoS One 2014;9:e90126.

49 Li L, Liu Y, Wu P, et al. Influenza-associated excess respiratory mortality in China, 2010-15: a population-based study. Lancet Public Health 2019;4:e473-81. 